\title{
COMMENTATIONES IN MEMORIAM ROLF NEVANLINNA
}

Rolf Nevanlinna died in Helsinki on the 28th May 1980 at the age of 84 . The 10th Rolf Nevanlinna Colloquium, planned to be held in Zürich in the autumn of 1980 on the occasion of his 85 th birthday, was postponed and became a memorial conference. Organized by Kurt Strebel and Hans Künzi it was held at the University of Zürich on 5-6 March 1981. The program consisted of lectures describing Nevanlinna's mathematical work and its later developments.

The present issue contains all these lectures, in the order they were given in the Colloquium:

Lehto, Olli, On the birth of the Nevanlinna theory

Carleson, Lennart, Estimates of harmonic measures

Fuchs, W. H. J., The development of the theory of deficient values since Nevanlinna

Ahlfors, Lars V., Riemann surfaces and small point sets

Toppila, Sakari, On Nevanlinna's proximity function

Hayman, W. K., Some achievements of Nevanlinna theory

Pfluger, Albert, Über konforme Abbildungen des Einheitskreises

Rickman, Seppo, Value distribution of quasimeromorphic mappings

Wittich, H., Anwendungen der Wertverteilungslehre auf gewöhnliche Differentialgleichungen

Bleuler, Konrad, Differentialgeometrische Methoden in der Physik.

Besides, there is a list, prepared by Ilppo Simo Louhivaara, of Rolf Nevanlinna's publications after 1976 .

Helsinki, May 1981

Olli Lehto 